\title{
Disseminated Eruptive Blue Nevi in a Young Adult Patient
}

Hasina Maredia ${ }^{1}$, Amarachi Eseonu ${ }^{1}$, Sima Rozati $^{1}$

1. Department of Dermatology, Johns Hopkins University School of Medicine, Baltimore, USA

Corresponding author: Hasina Maredia, hasinamaredia@gmail.com

\begin{abstract}
Common blue nevi tend to be singular or localized, with multiple eruptive blue nevi being a rare occurrence. We report the case of a young adult who presented with multiple asymptomatic lesions that had appeared gradually over a few years. Physical examination revealed 30 distinct, blue-gray macules diffusely over the medial buttocks, lower back, and dorsal arms. Histopathology showed pigmented dendritic melanocytes with associated melanophages, features characteristic of blue nevus. This case demonstrates that eruptive blue nevi can present as numerous, disseminated lesions over multiple anatomic sites. Recognition of the various patterns of eruptive blue nevi and their benign nature can reduce unnecessary biopsies and work-up.
\end{abstract}

Categories: Dermatology, Pathology

Keywords: eruptive blue nevi, benign nevi, melanocytes

\section{Introduction}

Common blue nevi occur in about $0.5-4.0 \%$ of healthy white adults, while multiple eruptive blue nevi are rarer and have been described in only 13 case reports so far [1-7]. These cases were associated with a known or possible trigger, and only two of them had multiple areas of the body involved $[2,6]$. We present a unique case of disseminated, discrete eruptive blue nevi over multiple anatomic sites to highlight a rare but benign presentation.

\section{Case Presentation}

A young adult presented to dermatology with multiple, discrete round small blue-gray macules that had first appeared a few years ago. The patient was otherwise healthy except for a history of scoliosis. The macules had initially appeared on the bilateral buttocks and then on the lower back and arms. The lesions were asymptomatic and there was no reported trauma to the area nor any tanning-bed use. There was no family history of similar lesions. On exam, 30 distinct, blue-gray, and well-demarcated macules, ranging in size from $1 \mathrm{~mm}$ to $3 \mathrm{~mm}$, were found on the medial buttocks (Figure 1), lower back, and bilateral dorsal arms. A punch biopsy was performed, and histopathology revealed pigmented dendritic melanocytes with associated melanophages, consistent with blue nevus (Figure 2). 


\section{Cureus}

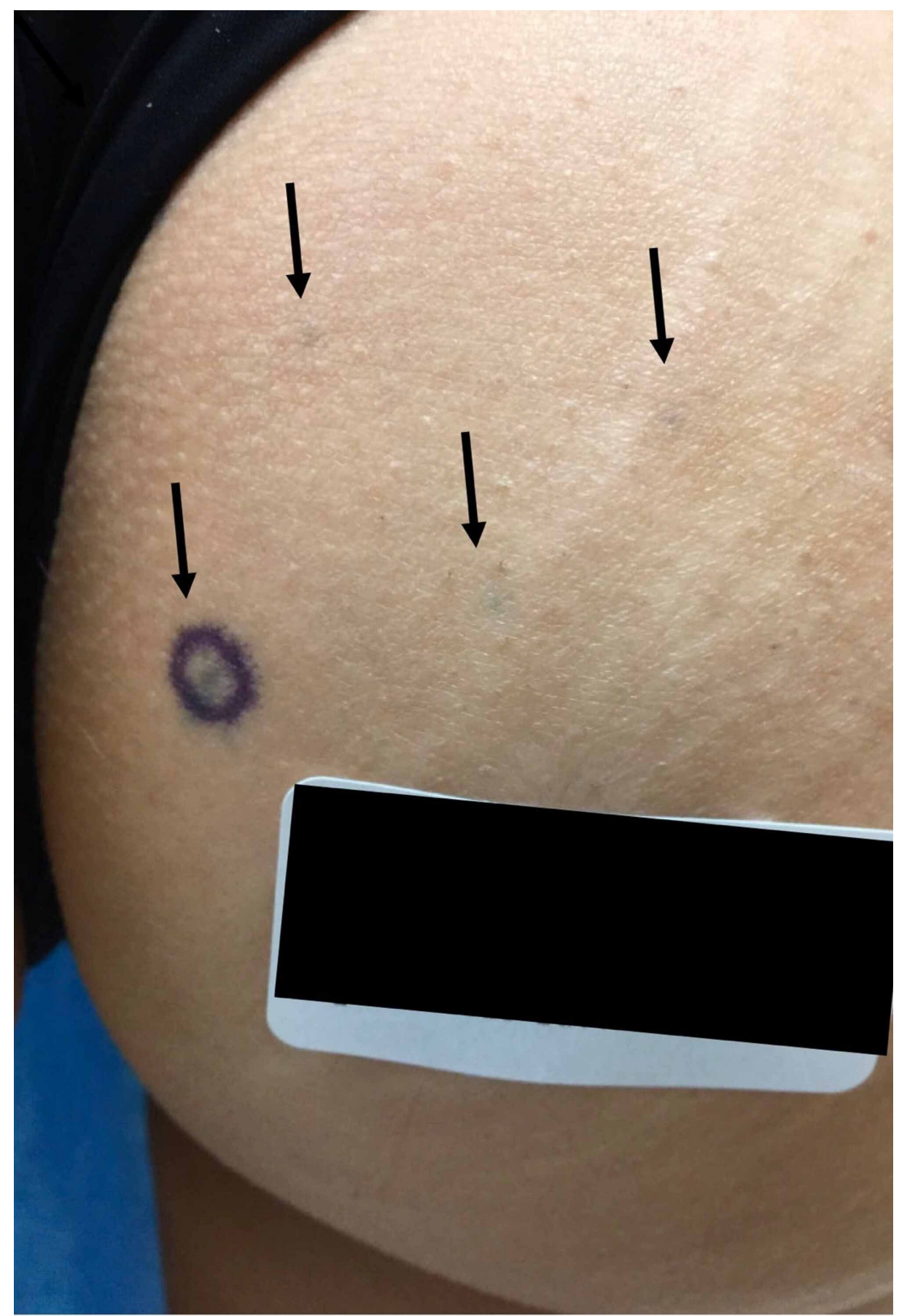

FIGURE 1: Clinical image of the right buttock demonstrating blue-gray macules

Four 1-3 mm, blue-gray, and well-demarcated macules on the right medial buttock pictured. The patient had additional similar macules on the left buttock, lower back, and bilateral dorsal arms, totaling around 30 macules 


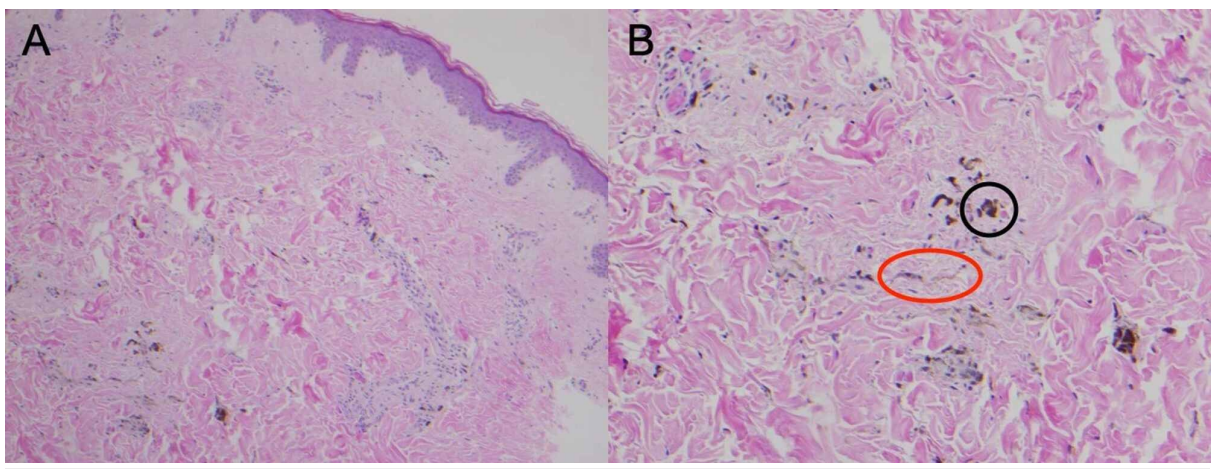

FIGURE 2: Punch biopsy specimen from lesion on the right buttock

III-defined, deep dermal proliferation of pigmented dendritic melanocytes (red circle) with associated melanophages (black circle). No associated mitoses or necrosis were appreciated, consistent with common blue nevus. Hematoxylin-eosin (A) x10 (B) x20

\section{Discussion}

Diagnosis of blue nevi can be made clinically, although atypical and challenging cases may require a biopsy to confirm the diagnosis. Histologically, the blue nevi are located in the mid-dermis and are thought to arise from dendritic melanocytes that have not migrated to the epidermis from their site of origin in neural crest cells [1-4]. They commonly present in areas with residual dermal melanocytes, including the scalp, presacrum, and dorsal extremities [5-7].

Types of blue nevi, based on clinical and histopathological differences, include common and cellular [1-3]. Common blue nevi tend to be solitary blue-black papules, less than $10 \mathrm{~mm}$ in size. Histologically, they appear as an admixture of pigmented dendritic melanocytes and melanophages in a fibrotic stroma [3]. In contrast, cellular blue nevi are firm, blue-black, and dome-shaped nodules or plaques that tend to be $1-2 \mathrm{~cm}$ in size but can be larger [5]. Histologically, cellular blue nevi are characterized by distinct cellular areas with spindled-to-oval-shaped melanocytes and clear-to-lightly pigmented cytoplasm [3].

Among the 13 cases of eruptive blue nevi reported so far, only two had multiple areas involved, namely a patient with bilateral tibial involvement and a second with diffuse distribution over the body $[2,6]$. However, both had a history of additional cutaneous lesions. The former had cutis marmorata telangiectatica congenita along with limb hypoplasia [6]. The latter had generalized congenital dermal melanocytosis and acquired bilateral nevus of Ota, and the patient's blue nevi erupted after starting tanning-bed use and oral contraceptives [2]. In comparison, our patient presented uniquely with a disseminated distribution of discrete multiple blue nevi and no associated lesions.

The cases of eruptive blue nevi were associated with a known or possible trigger, although there may have been a selection bias for reporting such cases. Triggers or associations have included trauma, sunburns, oral contraceptives, and vesiculobullous dermatoses [1-3]. In addition, multiple blue nevi may also be associated with the lentigines, atrial myxomas, mucocutaneous myxomas, and blue nevi (LAMB) syndrome [3]. Our patient did not have any identifiable trigger or association.

It is important to distinguish blue nevi from satellite metastatic melanoma or regressive melanoma [4]. Common blue nevi are stable and can regress, though there have been rare reports of malignant blue nevi, mostly with the cellular type [3]. Thus, the presence of nodules or changes in the appearance of blue nevi warrants work-up. Other differentials for blue nevi include glomus tumor, Spitz nevi, sclerosing hemangioma, Kaposi sarcoma, and dermatofibroma [2]. Atypical acquired dermal melanocytoses are also on the differential, but a review of 15 such cases found that they occur mainly over the face, extremities, and the back with only three cases that had melanophages on histology [8]. Thus, our case was most consistent clinically and histologically with eruptive blue nevi.

\section{Conclusions}

Given limited data in the literature, our case report provides an example of eruptive blue nevi that can be benign even when numerous, disseminated over multiple anatomic sites, and sudden in appearance. The recognition of unique, benign patterns can reduce unnecessary biopsies and provide reassurance to patients. Further long-term follow-up studies are needed to truly assess the risk of malignant transformation.

\section{Additional Information}

\section{Disclosures}


Human subjects: Consent was obtained by all participants in this study. Conflicts of interest: In compliance with the ICMJE uniform disclosure form, all authors declare the following: Payment/services info: All authors have declared that no financial support was received from any organization for the submitted work. Financial relationships: All authors have declared that they have no financial relationships at present or within the previous three years with any organizations that might have an interest in the submitted work. Other relationships: All authors have declared that there are no other relationships or activities that could appear to have influenced the submitted work.

\section{References}

1. Colson F, Arrese JE, Nikkels AF: Localized eruptive blue nevi after herpes zoster . Case Rep Dermatol. 2016, 8:118-123. 10.1159/000446178

2. Diehl JW, Berk DR, Ney A, Bayliss SJ: Diffuse dermal melanocytosis: follow-up 30 years later with novel findings of eruptive blue nevi. Arch Dermatol. 2011, 147:1339-1340. 10.1001/archdermatol.2011.308

3. Zembowicz A, Phadke PA: Blue nevi and variants: an update. Arch Pathol Lab Med. 2011, 135:327-336. 10.1043/2009-0733-RA.1

4. Zembowicz A: Blue nevi and related tumors. Clin Lab Med. 2017, 37:401-415. 10.1016/j.cll.2017.05.001

5. Kim YJ, Cohen PR: Blue nevus developing at a combined tetanus, diphtheria, and pertussis (Tdap) vaccination site: case report and summary of vaccine-associated cutaneous lesions. Cureus. 2019, 11:e4997. 10.7759/cureus.4997

6. Krause MH, Bonnekoh B, Weisshaar E, Gollnick H: Coincidence of multiple, disseminated, tardive-eruptive blue nevi with cutis marmorata teleangiectatica congenita. Dermatology. 2000, 200:134-138. 10.1159/000018347

7. Kesty K, Zargari O: Eruptive blue nevi. Indian J Dermatol Venereol Leprol. 2015, 81:198-201. 10.4103/03786323.152302

8. Harrison-Balestra C, Gugic D, Vincek V: Clinically distinct form of acquired dermal melanocytosis with review of published work. J Dermatol. 2007, 34:178-182. 10.1111/j.1346-8138.2007.00245.x 